\title{
Translação do conhecimento produzido em um grupo de pesquisa: uma reflexão
}

\section{discente}

\author{
Translation of knowledge produced in a research group: a student reflection
}

Traducción de conocimientos producidos en un grupo de investigación: una reflexión del estudiante

Fabieli Borges

ORCID: https://orcid.org/0000-0002-0835-5311 Universidade Federal do Paraná, Brasil E-mail: fabieliborges6@gmail.com

Olívia Luciana dos Santos Silva ORCID: https://orcid.org/0000-0002-5927-0238 Universidade Federal do Paraná, Brasil E-mail: olivialucianas@ gmail.com

Luis Fernando Gualdezi

ORCID: https://orcid.org/0000-0001-9737-5690 Universidade Federal do Paraná, Brasil

E-mail: gualdezi@icloud.com

Karla Crozeta Figueiredo

ORCID: https://orcid.org/0000-0003-3544-5643 Universidade Federal do Paraná, Brasil

E-mail: karla.crozetafigueiredo@gmail.com

Elizabeth Bernardino

ORCID: https://orcid.org/0000-0003-1321-8562 Universidade Federal do Paraná, Brasil

E-mail: elizaber@ufpr.br

Aida Maris Peres

ORCID: https://orcid.org/0000-0003-2913-2851 Universidade Federal do Paraná, Brasil E-mail: aidamaris.peres@gmail.com

\begin{abstract}
Resumo
A articulação da teoria e prática é uma necessidade crescente na práxis da Enfermagem. É neste ensejo que a translação do conhecimento precisa ser considerada na formação deste profissional. Os grupos de pesquisa da pósgraduação, por exemplo, configuram-se um espaço em potencial para esta iniciativa. Objetivo: Refletir como o conhecimento produzido em um grupo de pesquisa contribuiu para a formação e qualificação profissional de enfermeiros à luz da translação do conhecimento. Método: Trata-se de um artigo de reflexão que retrata a vivência de pós-graduandos de enfermagem membros de um grupo de pesquisa de um Programa de Pós-graduação de Enfermagem da Universidade Federal do Paraná. Estas vivências giram em torno do conhecimento produzido mediante participação no grupo e a contribuição deste para a formação profissional. Resultados: A vivência do grupo foi representada pelas categorias: "Impacto do conhecimento produzido no GPPGPS para a dimensão ensinar/educar do enfermeiro" e, "Impacto do conhecimento produzido no GPPGPS para a dimensão cuidar do enfermeiro". Posteriormente, o conhecimento produzido no grupo de pesquisa foi representado no Modelo para a translação (ou aplicação) do conhecimento, atendendo às suas etapas. Considerações finais: Esta reflexão sob olhar da TC, permitiu visualizar como houve o impacto deste conhecimento produzido no grupo de pesquisa por meio de cada etapa do ciclo de ação. O grupo de pesquisa é um espaço de discussão acadêmica responsável pela emersão de resultados que podem transformar uma prática profissional com impacto para a prática de enfermagem em suas várias frentes, para a saúde e sociedade.
\end{abstract}

Palavras-chave: Programas de pós-graduação em saúde; Enfermagem; Translação do conhecimento; Disseminação da informação; Ensino.

\footnotetext{
Abstract

The articulation of theory and practice is a growing need in nursing practice. It is in this context that the translation of knowledge needs to be considered in the training of this professional. Graduate research groups, for example, are a potential space for this initiative. Objective: To reflect on how the knowledge produced in a research group contributed to the training and professional qualification of nurses in light of the translation of knowledge. Method: This is a reflection article that portrays the experience of nursing graduate students who are members of a research group of a Nursing Graduate Program at the Federal University of Paraná. These experiences revolve around the knowledge produced through participation in the group and its contribution to professional training. Results: The
} 
experience of the group was represented by the categories: "Impact of the knowledge produced in the GPPGPS for the teaching/educating dimension of the nurse" and "Impact of the knowledge produced in the GPPGPS for the dimension of caring for the nurse". Subsequently, the knowledge produced in the research group was represented in the Model for the translation (or application) of knowledge, taking into account its stages. Final consideration: This reflection from the perspective of CT, allowed us to visualize how this knowledge produced in the research group had an impact through each stage of the action cycle. The research group is a space for academic discussion responsible for the emergence of results that can transform a professional practice with an impact on nursing practice on its various fronts, on health and society.

Keywords: Health postgraduate programs; Nursing; Knowledge translation; Dissemination of information; Teaching.

\section{Resumen}

La articulación de la teoría y la práctica és una necesidad creciente en la práctica de la enfermería. És en este contexto que la traducción de conocimientos debe ser considerada en la formación de este profesional. Los grupos de investigación de posgrado, por ejemplo, son un espacio potencial para esta iniciativa. Objetivo: Reflexionar sobre cómo el conocimiento producido en un grupo de investigación contribuyó a la formación y calificación profesional del enfermero a la luz de la traducción del conocimiento. Método: Este és un artículo de reflexión que retrata la experiencia de estudiantes de posgrado en Enfermería que son miembros de un grupo de investigación de un programa de posgrado en enfermería de la Universidade Federal do Paraná. Estas experiencias giran en torno al conocimiento producido por la participación en el grupo y su contribución a la formación profesional. Resultados: La experiencia del grupo estuvo representada por las categorías: "Impacto del conocimiento producido en la GPPGPS para la dimensión docente / educativa del enfermero" e "Impacto del conocimiento producido en el GPPGPS para la dimensión del cuidado del enfermero". Posteriormente, el conocimiento producido en el grupo de investigación fue representado en el Modelo de traducción (o aplicación) de conocimiento, teniendo en cuenta sus etapas. Consideraciones finales: Esta reflexión desde la perspectiva del PC, permitió visualizar cómo este conocimiento producido en el grupo de investigación incidía en cada etapa del ciclo de acción. El grupo de investigación és un espacio de discusión académica responsable de la emergencia de resultados que puedan transformar una práctica profesional con impacto en la práctica de enfermería en sus distintas frentes, en la salud y la sociedad.

Palabras clave: Programas de posgrado en salud; Enfermería; Traducción de conocimientos; Diseminación de información; Enseñanza.

\section{Introdução}

O ano de 2020 foi escolhido pela Organização Mundial de Saúde como o ano Internacional dos profissionais de Enfermagem para homenagear os 200 aniversários de Florence Nightingale (World Health Organization, 2020). Foi marcado também por mudanças no modo de ensinar, aprender e praticar enfermagem, pois a pandemia do COVID-19 reinventou o modo de cuidar e de viver. Os sistemas de saúde lidam com o desafio constante de melhorar a qualidade dos cuidados e diminuir o risco de tratamentos inadequados ou insuficientes, enquanto se mantém economicamente viáveis (Vieira et al., 2020).

Com a pandemia novos desafios foram impostos ao mundo. Aos profissionais de saúde, o conhecimento científico tornou-se ordem do dia. No entanto, em geral são necessários cerca de 17 anos para traduzir 14\% das pesquisas originais em benefícios para os usuários. Assim, diante do atual cenário de saúde, faz necessário relevar estratégias que impulsionam intervenções pautadas em conhecimento pertinente. Com isso, há de se pensar a importância das conexões entre os desafios da saúde global e as estratégias que articulem ciência e realidade objetiva (Zepeda et al., 2018).

O estudo acerca da Translação de Conhecimento vem ganhando os espaços acadêmicos face à aos cenários de saúde. Contudo, os estudos entornam ainda a difusão conceitual e a projeção para a realidade brasileira e local fortalecendo a translação do conhecimento como uma necessidade e quanto um desafio. (Ferreira \& Tavares, 2021).

Os grupos de pesquisa em enfermagem surgem, neste contexto, como mais uma opção pedagógica que a academia oferece na busca do aumento e melhoria da produção científica (Azevedo et al., 2018). Sendo, com isso, espaço oportuno para influenciar o desempenho do capital humano para o fortalecimento da gestão do conhecimento (Silva et al., 2018).

Vieira et al. (2020) apontam que, tradicionalmente, a tendência dos acadêmicos é de transferir ou difundir conhecimento, o que frequentemente significa publicar e apresentar os resultados dos estudos em eventos. Contudo, o conceito 
da TC oferece uma proposta muito mais ambiciosa direcionada para mudanças concretas na prática. Definido por Graham (2006, p.1) a TC é um "processo dinâmico e interativo que inclui a síntese, disseminação, intercâmbio e aplicação eticamente sólida de conhecimento para melhorar, proporcionar produtos e serviços mais efetivos e fortalecer o sistema de saúde".

Este estudo surgiu de uma produção como requisito para avaliação da Disciplina Integração do Conhecimento das Linhas de Pesquisa. Com a formação do grupo de trabalho e discussão coletiva, percebeu-se que os enfermeiros atuavam em distintas frentes profissionais e, com isso, surgiu a necessidade de visibilizar a experiência vivenciada por cada um deles no grupo de pesquisa e como essa vivência impacta/impactou em sua formação e/ou qualificação profissional. A lacuna para este estudo incorre no TC como um tema crescente, porém, com exemplos da prática ainda pouco difundidos. Assim, a partir da experiência descrita, utilizou-se a TC como um suporte teórico para emergir as reflexões em torno dos impactos do grupo de pesquisa na perspectiva discente, para a práxis da Enfermagem.

Ante ao exposto, apresenta-se a questão norteadora deste estudo: Como o conhecimento produzido no Grupo de pesquisa pode impactar na formação e qualificação profissional? E, como objetivo tem-se: Refletir como o conhecimento produzido em grupo de pesquisa contribuiu para a formação e qualificação profissional de enfermeiros à luz da Translação do Conhecimento.

\section{Metodologia}

Trata-se de um estudo do tipo teórico-reflexivo a qual utilizou da descrição de experiências vivenciadas pelos próprios pesquisadores como discentes da pós-graduação inseridos em grupos de pesquisa. As reflexões foram apresentadas à luz do referencial teórico proposto por Graham (2006) acerca da Translação do Conhecimento e o modelo proposto pelo Canadian Institutes of Health Research (2012).

Este estudo é oriundo de uma atividade avaliativa de uma disciplina intitulada "Integração do Conhecimento das Linhas de Pesquisa, ofertada pelo Programa de Pós-graduação em Enfermagem da Universidade Federal do Paraná (PPGEnf/UFPR). A disciplina aconteceu de forma remota via Plataforma Microsoft Teams nos meses de maio a julho de 2020, com um total de sete encontros em formato de reuniões síncronas e de atividades orientadas.

Entre seus procedimentos didáticos, a disciplina previa a elaboração de um produto de modo a retratar o grupo de pesquisa como espaço potencializador de construção do conhecimento. Houve uma divisão dos discentes matriculados, com a sugestão de serem alunos sob diferentes docentes orientadores e na disposição de grupos.

O grupo foi formado por três pós-graduandos do PPGEnf/UFPR (um mestrando e duas doutorandas). Em discussão coletiva, optou-se pela elaboração de um manuscrito que englobasse a temática: Impactos do conhecimento produzido para a formação e qualificação profissional, ora direcionado pela docente da disciplina.

Diante da temática prevista, houve encontros virtuais síncronos para apropriação da mesma. Assim, num primeiro momento, foi elencado como critério, incluir a experiência descrita pelo discente limitando-se à participação no Grupo de Pesquisa em Políticas, Gestão e Práticas em Saúde (GPPGPS) do PPGEnf/UFPR, uma vez membros deste. O GPPGPS está ativo no PPGEnf/UFPR desde o ano de 2008 e estrutura-se em dois grandes projetos/linhas: Gerenciamento de ações e serviços de Saúde e Enfermagem e, Políticas, práticas de saúde e de Educação em Enfermagem.

As experiências descritas pelos discentes entornaram a reflexão: "como participar de um grupo de pesquisa contribuiu para a minha formação e qualificação profissional no meu local de trabalho?". A experiência foi descrita e digitalizada em documento Microsoft Word, individualmente, contemplando temas das quais participou e outros aspectos que o discente julgasse pertinente. Posteriormente, o grupo reuniu as descrições e as apresentou em duas categorias, a saber: "Impacto do conhecimento produzido no GPPGPS para a dimensão ensinar/educar do enfermeiro" e, "Impacto do conhecimento produzido no GPPGPS para a dimensão cuidar do enfermeiro". 
A partir do quadro, sentiu-se a necessidade de aprofundar a análise destas experiências descritas incluindo um referencial teórico para fortalecer e fornecer um "óculos" para a reflexão. Por meio do referencial teórico da TC, a análise percorreu as etapas do Conhecimento-para-Ação (Graham et al., 2006 \& Canadian Institutes of Health Research, 2012), articulando o conhecimento adquirido no grupo de pesquisa e como se projetou na prática.

Por configurar-se um estudo do tipo reflexivo, com base em experiências vivenciadas na perspectiva de discentes, não foi necessário o registro no sistema CEP/CONEP.

\section{Resultados e Discussão}

As experiências descritas e vivenciadas pelos discentes, estão apresentadas no Quadro 1 que segue. É possível perceber que os mesmos estão lotados em cenários de trabalho que envolvem a dimensão ensinar/educar e cuidar do enfermeiro.

Quadro 1 - Descrição da experiência por meio dos relatos dos membros do Grupo de pesquisa dispostas em categorias.

Curitiba, PR, 2020.

\begin{tabular}{|c|c|}
\hline CATEGORIA & DESCRIÇÃO DA EXPERIÊNCIA \\
\hline $\begin{array}{l}\text { Impacto do conhecimento } \\
\text { produzido no GPPGPS para a } \\
\text { dimensão ensinar/educar do } \\
\text { enfermeiro }\end{array}$ & 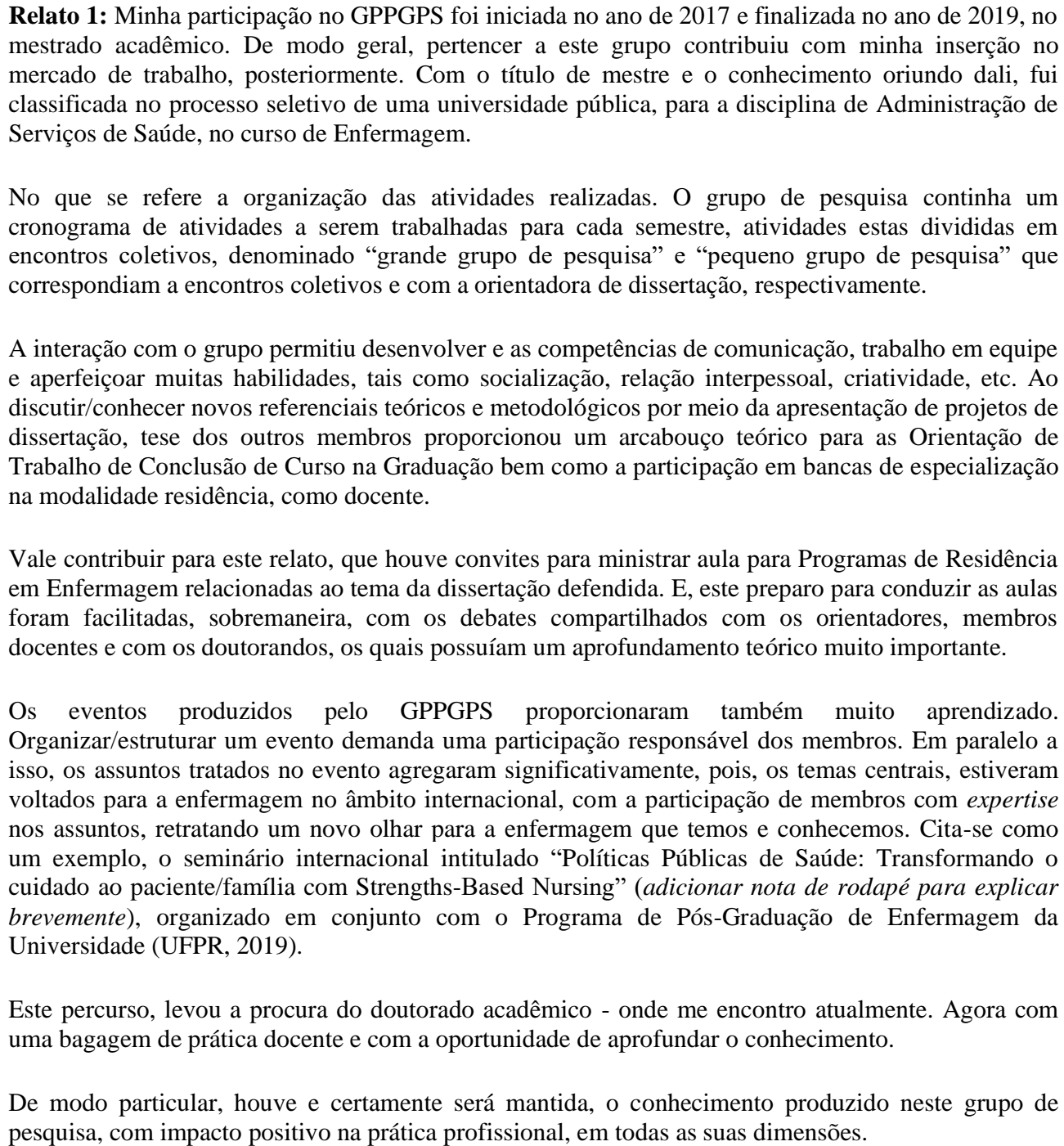 \\
\hline
\end{tabular}




\begin{tabular}{|c|c|}
\hline & $\begin{array}{l}\text { Relato 2: Meu ingresso no GPPGPS ocorreu no ano de } 2017 \text {, enquanto acadêmico do curso de } \\
\text { enfermagem de uma instituição privada na cidade de Curitiba - PR. Inicialmente minhas participações } \\
\text { eram de forma observacional, com o objetivo de agregar conhecimento sobre pesquisa e ensino em } \\
\text { enfermagem. Comecei a participar ativamente do Grupo em } 2018 \text {, quando realizei meu trabalho de } \\
\text { conclusão de curso. Ali pude apresentar ao Grupo e discutir a forma que conduziria a pesquisa, os } \\
\text { elementos metodológicos e a coleta de dados. Durante esse período, as contribuições ocorreram de } \\
\text { forma substancial, no que tange o desenvolvimento dessas atividades. } \\
\text { No ano de } 2019 \text { ingressei no mestrado acadêmico em enfermagem, no qual minhas participações } \\
\text { tornaram-se mais ativas no grupo de pesquisa. O grupo possui um calendário variado com a } \\
\text { participação dos seus integrantes em eventos, na elaboração de resumos, apresentação de pôsteres, na } \\
\text { apresentação pública de teses e dissertações e na produção de artigos científicos. Participar do grupo } \\
\text { de pesquisa possibilita enxergar os desafios e apropriar-se de conhecimentos e habilidades necessárias } \\
\text { ao desenvolvimento das atividades propostas. } \\
\text { A presença de acadêmicos de enfermagem nos grupos de pesquisa é de suma relevância, pois } \\
\text { possibilita um olhar diferenciado nas propostas de trabalho da enfermagem. Dentre elas podemos } \\
\text { destacar o compartilhamento de vivências relativas à prática profissional na assistência de } \\
\text { enfermagem, da pesquisa e do ensino e, claro entrelaçados ao gerenciamento. }\end{array}$ \\
\hline $\begin{array}{l}\text { Impacto do conhecimento } \\
\text { produzido no GPPGPS para a } \\
\text { dimensão cuidar do enfermeiro }\end{array}$ & $\begin{array}{l}\text { Relato 3: Meu contato com grupos de pesquisa vem, como uma parcela dos estudantes de } \\
\text { enfermagem, desde a graduação. Formada há } 14 \text { anos, em uma universidade pública do Nordeste, } \\
\text { cursei especialização e mestrado em universidades da região sudeste. Atualmente, devido ao ingresso } \\
\text { em serviço público como enfermeira assistencial, estou cursando o doutorado em uma universidade } \\
\text { pública do sul do Brasil. } \\
\text { A vivência mais recente em grupos de pesquisa é também a mais marcante e intensiva. Integrante do } \\
\text { GPPGPS, desde o ano passado, tive a oportunidade de, agora, conectar a pesquisa com a minha } \\
\text { prática assistencial; bem como levar um pouco da prática para o ambiente acadêmico. } \\
\text { As reuniões eram momentos de socialização únicos. O reencontro com colegas - alguns também } \\
\text { colegas de trabalho - era momento de dividir experiências acadêmica ou assistencial. Compartilhamos } \\
\text { ansiedades pela incompreensão de um tema ou trabalho por fazer; porém, nesse mesmo espaço } \\
\text { surgiam as resoluções e ajudas, que em discussão no grupo, pareciam fáceis e simples. } \\
\text { A realização de trabalhos em grupo, com a sugestão de ser com colegas de diferentes orientadores, } \\
\text { foram momentos enriquecedores. Como na assistência, conviver com a diferença - de tema, de } \\
\text { orientador - foi um desafio que o grupo, no transcorrer do trabalho, vence com propriedade. } \\
\text { No que tange aos temas apresentados, em grande parte como produto da pesquisa da pós-graduação, } \\
\text { envolvem assuntos relevantes para a assistência de enfermagem. No meu caso, como trabalho como } \\
\text { supervisora em uma unidade de internação, os principais conceitos em gestão e gerenciamento são } \\
\text { atualizados constantemente. }\end{array}$ \\
\hline
\end{tabular}

Fonte: Autores (2020).

Mediante categorias oriundas dos relatos, é possível refletir a construção do conhecimento à luz da Translação, isto, conectando a teoria e a prática. O Modelo de TC proposto pelo Canadian Institutes of Health Research (2012) contempla um ciclo de desenvolvimento do conhecimento. Este ciclo é ilustrado pelo funil, apresentado no centro da figura, que refina o conhecimento e também, o ciclo de ação externo ilustrando o processo de aplicação do conhecimento representado pela figura. Destaca-se que as sete fases do ciclo de ação que são dinâmicas e interdependentes. Estas, foram desenvolvidas baseadas em teorias de ações planejadas, no intuito de auxiliar o gestor de modo didático e prático a aplicar o conhecimento em prática (Graham, 2006; Tetroe, 2007). 
Figura 1 - Modelo para a Translação (ou aplicação) do Conhecimento.



Fonte: Canadian Institutes of Health Research (2012).

Para a melhor compreensão dos impactos gerados pelo conhecimento adquirido/ vivenciado no grupos de pesquisa e relatados neste estudo, foi seguido os sete itens do ciclo- de- ação, proposto no modelo acima: a. identificar um problema que precisa ser abordado: identificar, analisar e selecionar o conhecimento ou a pesquisa relevante para a necessidade; b. adaptar o conhecimento ao contexto local; c. identificar os fatores favoráveis e as barreiras para o uso do conhecimento: selecionar as estratégias mais apropriadas; d. implementar a mudança; e. monitorar o uso do conhecimento; f. avaliar os resultados da utilização do conhecimento; g. apoiar o uso contínuo do conhecimento no intuito de mantê-lo (Canadian Institutes of Health Research, 2012, tradução nossa).

Este processo é interativo e dinâmico, possuindo limites flexíveis entre componentes da criação do conhecimento e da ação (Graham et al, 2006). Isto é, por mais que se inicie na identificação do problema, há uma movimentação entre uma etapa e outra bem como a possibilidade de retornar a etapa anterior, visando a viabilidade do uso do conhecimento (Oelke, Lima \& Acosta, 2015). Isto remete à reflexão de que, em nossa prática, por vezes, é preciso avaliar e reavaliar os processos propostos para a prática e, por vezes, voltar à estas.

Considerando a descrição da experiência vivenciada no grupo de pesquisa, pode-se visualizar o impacto do conhecimento produzido à luz da TC. Os principais elementos foram identificados e projetados no modelo de translação do conhecimento (Figura 2). 
Figura 2 - Conhecimento produzido no grupo de pesquisa representado no Modelo para a translação (ou aplicação) do

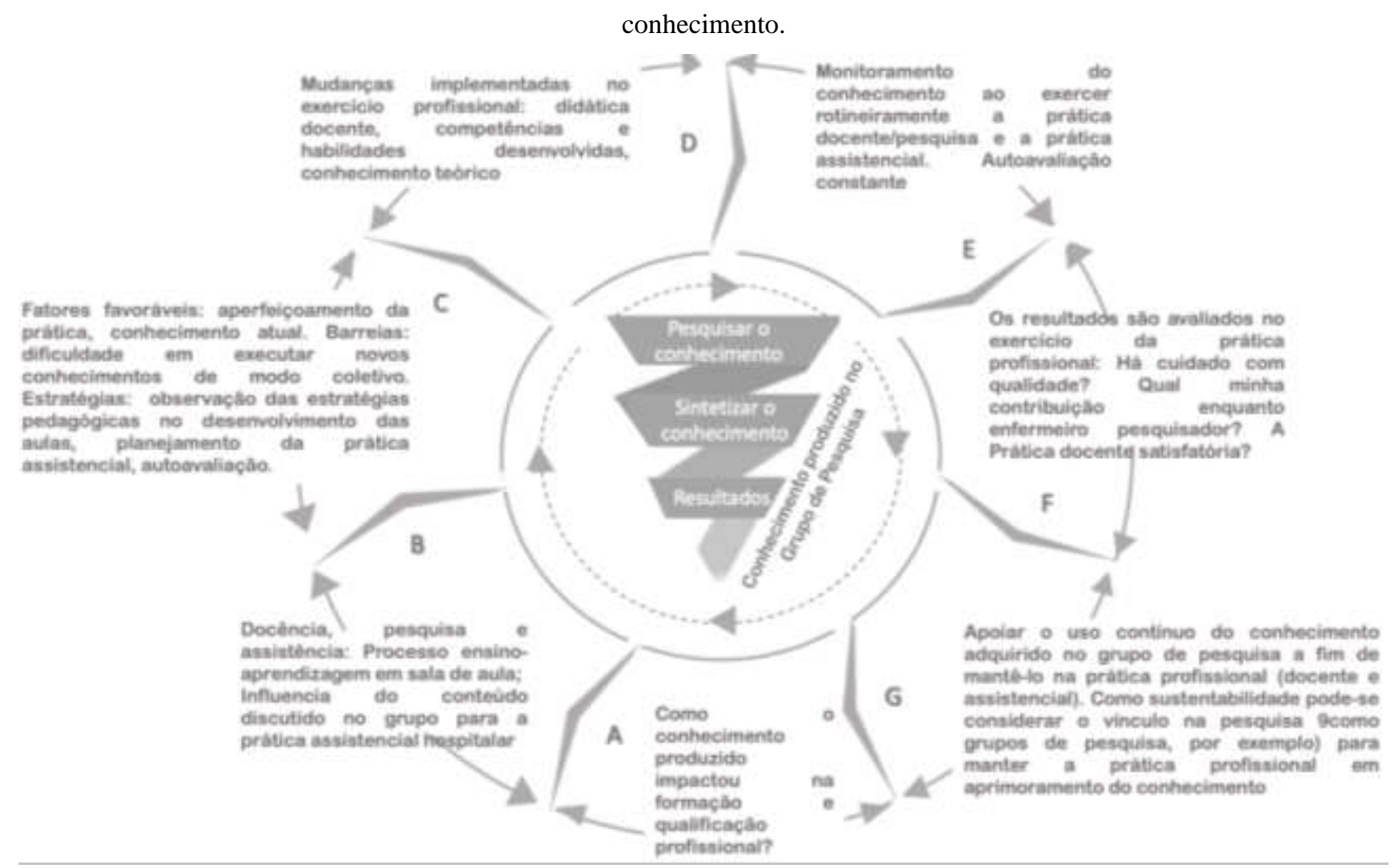

Fonte: Adaptado de Canadian Institutes of Health Research (2012).

Dos grupos de pesquisas, organizados por eixos temáticos ou linhas de pesquisa, emergem a produção de pesquisa, tendo por base às lacunas do conhecimento em saúde. Trata-se do objetivo central dos programas de pós-graduação na enfermagem, expressados num aumento nacional significativo no longo dos anos, agregando na produção científica bem como na prática assistencial (Crosseti \& Gôes, 2017). É este o sentido de o centro da figura apresentar o grupo de pesquisa e o conhecimento adquirido nele. É claro, que ampliando o olhar, é preciso sim considerar a Universidade/graduação também com este importante papel de formação do profissional com implicações na sua prática de trabalho. Contudo, na percepção dos pesquisadores discentes desta reflexão, a pós-graduação e a sua inserção no grupo parece "despertar" o enfermeiro para situações da prática.

O levantamento documental de Ferreira \& Tavares (2021), por meio dos sites dos cursos, trabalhos de conclusão e os produtos de um programa de pós-graduação de mestrado profissional em enfermagem, apontou a inexistência de menção e/ou referência de modelo orientador para a TC, embora informações nos referidos material configurem uma perspectiva translacional. Os autores concluem que no Brasil, a TC configura-se uma necessidade e em paralelo, é desafiador no campo da pesquisa. É destaque a urgência na adaptação de modelos teóricos para a realidade brasileira. Isto incorre na ideia de academia e prática parceiras em se apropriar em estudos que avaliem, validem e implementem mudanças.

Em complemento à ideia anterior, a reflexão de Vieira, Gastaldo \& Harisson (2020) aponta que, dado as experiências canadenses neste modelo teórico, é primordial a promoção de treinamento de pesquisadores e estudantes para desenvolver habilidades críticas de avaliação da qualidade das publicações cientificas para possível aplicação à pratica. Para os autores, o Brasil ainda é incipiente no que se refere a pareceria entre pesquisadores, profissionais, pacientes, familiares e instituições, os recursos financeiros públicos são escassos e o investimento em pesquisas desta natureza é baixo. 
Com vistas à necessidade de estruturas mais robustas viabilizando à incorporação dos resultados na prática, e consequentes benefícios para a equipe de saúde, pacientes e familiares, é que entra em cena a perspectiva translacional da pesquisa em enfermagem (Crosseti \& Gôes, 2017). Frente às reflexões, num primeiro momento, percebe-se os aspectos relacionados às frentes de trabalho dos pós-graduandos, tão logo remetidos às dimensões da enfermagem. Estas dimensões dizem respeito aos processos de trabalho assistir, pesquisar, ensinar, administrar e participar politicamente (Sanna, 2007).

Embora a disposição das descrições entornou as dimensões pesquisar, ensinar e cuidar nas categorias, entende-se que em dado momento elas se inter-relacionam e, somado a isto, ancoram-se na dimensão administrar ao considerar que o grupo de pesquisa é da linha de gerenciamento de enfermagem e os participantes do estudo o integram. Isto refuta a ideia errônea de enfermeiros que interpretam que gerenciar representa afastamento do cuidado direto ao paciente. Esta dicotomia deve ser rompida, pois o cuidado e a gerência são indissociáveis (Borges \& Silva, 2013; Ferreira et al., 2019).

Ao pensar cuidado de enfermagem fragmentadas em dimensões, é possível que implique na dificuldade do enfermeiro, para traduzir o conhecimento aprendido em teoria para a sua práxis, ao passo que associar tais dimensões propunha uma atuação holística por este profissional. A necessidade de mais estudos que elenquem quais são os fatores limitadores para a TC e debruçar-se em estratégias para sua minimização é um aspecto que oriunda desta reflexão e caminho para o seu uso consolidado.

Destarte, ainda que a dimensão do cuidado consista no cerne da profissão, o gerenciamento tem-se constituído importante no conjunto de estratégias racionalmente formuladas, em ações objetivas e subjetivas que impactam em melhores condições de assistência e de trabalho da equipe de enfermagem. A literatura apresenta, com evidências científicas as contribuições do gerenciamento de enfermagem hospitalar, como por exemplo, facilitar o desenvolvimento e organização do trabalho na perspectiva técnico-política e qualificar os processos produtivos (Ferreira et al., 2019).

Explanado esta vertente, à luz do referencial teórico da TC, foi possível a articulação no contexto conhecimento produzido no grupo de pesquisa versus impacto na formação e qualificação profissional, como bem representado esquematicamente. A primeira fase do ciclo, portanto, correspondeu a este tema como a necessidade identificada.

Uma característica básica da TC, diz respeito à interação entre pesquisadores e usuários, isto é, enfermeiras pesquisadores e enfermeiras da prática, respectivamente. Há ainda, os usuários que utilizarão tais conhecimentos gerados pela pesquisa para tomada de decisões que poderão ser base para políticas, programas e/ou práticas de saúde. Essa interação varia ainda, em intensidade, complexidade e nível de engajamento e qual é o contexto - fatores físicos, estruturais, organizacionais, sociais (Canadian Institutes of Health Research, 2012 \& Crosseti, Góes, 2019). Neste estudo, entende-se que esta interação ocorreu com os participantes nos seus papéis de pesquisadores e usuários, concomitantemente.

É esperado que os enfermeiros usem evidências de pesquisa na prática para melhorar os resultados do cliente e do sistema. O conhecimento produzido no grupo de pesquisa agregou primeiramente para os participantes, ao fornecer conteúdo que impactasse na qualificação e formação profissional para inserção no mercado de trabalho. Tão logo, o conhecimento foi adaptado para cada contexto de trabalho, a saber: na docência ensino superior; no campo da pesquisa e para a prática assistencial na área hospitalar.

Corrobora nesta perspectiva, uma revisão sistemática financiada pelos Institutos de Pesquisa Canadenses, que investigou e concluiu que as intervenções da TC direcionadas aos enfermeiros nos cuidados terciários, são eficazes para subsidiar as tomadas de decisões baseadas em evidências bem como promover os resultados dos clientes (Yost et al., 2015). Este mesmo estudo também se propôs a descobrir se o TC exerce influência na melhora dos conhecimentos, habilidades, comportamentos dos enfermeiros. Contudo, das 44.648 citações examinadas, não houve nenhum relato pelos enfermeiros acerca destes aspectos (Yost et al., 2015). Os achados provenientes deste estudo, em contrapartida, mencionam o desenvolvimento de competências e habilidades como conhecimento proveniente da participação do grupo de pesquisa. 
Competências e habilidades e aprofundamento de conhecimento teórico, como por exemplo, referenciais teóricos, metodologias, construção de artigos científicos foram descritas como importante reflexo da participação nos grupos de pesquisa. Os enfermeiros sempre são desafiados a como podem contribuir para a sociedade como profissionais, espera-se a responsabilidade pela prestação contínua de cuidados diretos, protegendo vidas individuais e apoiando atividades da vida diária. Para isso, é importante que os enfermeiros aprimorem sua competência em enfermagem e utilizem-na em sua prática diária. Competência é uma habilidade adquirida através da experiência e aprendizado (Fukada, 2018).

Os resultados dialogam ao que estabelece as Diretrizes Curriculares Nacionais de Enfermagem. A esses profissionais prima-se a necessidade de competências e habilidades, tais como atenção à saúde, tomada de decisões, comunicação, liderança, administração e gerenciamento, além de educação permanente. Tão logo, competências técnico-científicas, ético-políticas e sócio-educativas (Conselho Nacional da Educação, 2018).

Para a dimensão ensinar/pesquisar, a TC do conhecimento pode se configurar um importante alicerce para aprimoramento da prática. A experiência no magistério para o docente enfermeiro tem se configurado um espaço rico em formação oriundo dos conhecimentos gerados pelo modo como ele se apropria do 'ser professor' no decorrer da vida profissional. Ainda que tais saberes contribuam para o fortalecimento da profissão docente, eles não são suficientes para o exercício do magistério, haja vista que a função docente exige conhecimentos acadêmicos e competências técnicas que configuram um saber fazer que extrapola os processos de reprodução (Braga \& Boas, 2018).

Corrobora nesta perspectiva a mudança/aprimoramento em adquirir desenvoltura, domínio de conhecimento no que tange ao processo ensino-aprendizagem, caracterizado pela quarta fase do ciclo de ação. É oportuno acrescentar aqui, que a disciplina do programa, por ter ocorrido de forma remota emergencial, num contexto pandêmico, também aproximou os pósgraduandos do uso de tecnologias em torno da docência.

O aumento significativo de tecnologia inserido no contexto da saúde, demanda o repensar constante da enfermagem face à sua prática. A TC, por sua vez, tende a ser uma estratégia de ferramenta gerencial de qualificação do cuidado. Como exemplo, uma unidade neonatal de um hospital federal do Rio de Janeiro, utilizou da TC para implementar o processo de enfermagem no local. Como resultado, agregou melhorias dos registros de enfermagem e, possibilitou a qualificação dos cuidados prestados (Oliveira, Silvino \& Souza, 2021).

O uso do conhecimento deve ser mensurado, determinando sua difusão para os grupos alvos de usuários do conhecimento e considerando como ele está sendo utilizado e os resultados do seu uso (Grahram et al., 2006 \& Oelke et al., 2015). A importância na avaliação dos resultados é crucial para determinar a efetividade do plano de TC de modo a considerar resultados nos níveis do paciente, do profissional e do sistema. A complexidade de medir uma mudança não deve jamais ser subestimada (Oelke et al., 2015).

Neste ínterim, esta reflexão imbrica no pensamento do fazer enfermagem ao considerar cada etapa da TC. Reforça-se sobremaneira, a importância da linha de grupo de pesquisa, visibilizando suas produções. Recentemente, a Enfermagem na Gestão e Gerenciamento, foi uma das subáreas propostas na árvore do Conhecimento da Enfermagem em vigência. Isto porque as mudanças na ciência, ocorridas nas últimas três décadas, tiveram impacto no desenvolvimento do conhecimento na área de Enfermagem que, por sua vez, acompanhou as tendências mundiais. O reflexo disto, são os avanços necessários relacionados às ações de cuidado, de ensino e, consequente, às demandas de pesquisa na área de Enfermagem (Barros et al., 2020).

Esta perspectiva abre uma lacuna para estudos futuros que entornam, por exemplo, a identidade do grupo de pesquisa e de que forma, o cuidado traduzido para a prática implica nesta identidade. De que modo, a academia e a prática assistencial da enfermagem convergem? E, se divergem, o porquê? Como podem estreitar estes laços com vistas a melhorias de cuidados ao paciente, família, sociedade? A prática, seja ela de pesquisa ou do cuidar está satisfatória? 
Estas são algumas das indagações que emergiram ao refletir a contribuição do grupo de pesquisa para a formação e qualificação profissional. Espera-se que elas impulsionam para o aprofundamento deste novo olhar do enfermeiro para o seu saber e fazer lançando mão da TC na Enfermagem, enquanto profissão e enquanto disciplina.

\section{Considerações Finais}

Esta reflexão alcançou seu objetivo ao apresentar aspectos que caracterizam formação e qualificação profissional mediante a participação no GPPGPS em conexão à atuação profissional, isto é, na pratica docente, na pesquisa e na realidade hospitalar com competências e habilidades postas em práticas no mercado de trabalho, mas, com base fortalecida na academia.

O grupo de pesquisa constitui-se um espaço de discussão acadêmica responsável pela emersão de resultados que podem transformar uma prática profissional com impacto para suas várias frentes. Somado a isso, refletir as vivências oriundas deste espaço à luz da TC, implica numa prática transformadora para a oferta de serviços seguros e de qualidade, com implicações diretas para a sociedade.

Ao sistematizar o ciclo do conhecimento para a ação da TC, conduziu uma reflexão em torno do papel atribuído aos grupos de pesquisa. Estas, são unidades fomentadoras de saber, que amparam os participantes com diversos constructos. A partir dessa reflexão, é oportuno incitar que a enfermagem, em suas dimensões - gerência, ensino, pesquisa e assistência, pode se fortalecer como ciência ao criar espaços que promovam a TC. Espera-se que ao publicizar estas vivências, ancoradas neste importante referencial, seja também uma forma de divulgação dos impactos deste conhecimento para a práxis do enfermeiro bem como no incentivo de integração de enfermeiros da prática ao grupo, para o compartilhamento do saber e do fazer. Para as fragilidades identificadas no uso da TC na realidade brasileira, recomenda-se o fortalecimento de um trabalho de pesquisa em rede e a difusão nos campos de prática, tornando outras experiências visíveis.

Aponta-se como fator limitador para esta pesquisa, a escolha metodológica utilizada. A reflexão pautou-se em aspectos que entornaram a vivência de pós-graduandos de enfermagem, desse modo, limitou um olhar para atividades prevalentes neste contexto. Refletir no âmbito da TC, abriu um leque para pesquisas com técnicas diversas que a analisem qualitativamente e quantitativamente em cenários diversos da prática profissional do enfermeiro, alinhados às propostas de construção do conhecimento dentro e fora da academia.

\section{Referências}

Azevedo, I. C. et al. (2018). Importância do grupo de pesquisa na formação do estudante de enfermagem. Rev de Enferm da UFSM, 8(2): 390-398.

Barros, A. L. B. A et al. (2020). Pesquisa em enfermagem e a modificação da árvore do conhecimento no CNPq: contribuição à ciência. Rev. Bras. Enferm; 73(1): e20170911.

Borges, M. C. L. A \& Silva, L. M. S. (2013). (Des) articulação entre gerência e cuidado em uma unidade de terapia intensiva cirúrgica. $R$ Pesq: Cuid Fundam Online; 5(1):3403-3410.

Braga, M. J. G \& Bôas, L. V. (2018). Enfermagem e docência: uma reflexão sobre como se articulam os saberes do enfermeiro professor. Rev @ mbienteeducação; 7(2): $256-267$.

Canadian Institutes of Health Research (CIHR).(2012). Guide to Knowledge Translation Planning at CIHR: integrated and end-of-grant approaches. Canadá. www.cihr-irsc.gc.ca/e/documents/kt_lm_ktplan-en.pdf

Conselho Nacional de Educação. (2018). Câmara de Educação Superior. Resolução n. 543 de 31 de janeiro de 2018. Aprovar o Parecer Técnico n 28/2018 contendo recomendações do Conselho Nacional de Saúde (CNS) à proposta de Diretrizes Curriculares Nacionais (DCN) para o curso de graduação Bacharelado em Enfermagem, conforme anexo. Diário Oficial da União. Brasília. https://www.in.gov.br/materia//asset_publisher/Kujrw0TZC2Mb/content/id/48743098/do1-2018-11-06-resolucao-n-573-de-31-de-janeiro-de-2018-48742847

Crossetti, M. G. O; Góes, M. G. O. (2017). Translação do conhecimento: um desafio para prática de enfermagem. Rev Gaúcha Enferm;38(2):e74266.dx.

Ferreira, R. E \& Tavares, C. M. M. (2021). The perspective of knowledge translation in professional master's programs in nursing Research, Society and Development;10(11): e07101119168: 2525-3409. 
Research, Society and Development, v. 10, n. 17, e113101724071, 2021

(CC BY 4.0) | ISSN 2525-3409 | DOI: http://dx.doi.org/10.33448/rsd-v10i17.24071

Ferreira, V. H. S.; Teixeira, V. M.; Giacomini, M. A.; Alves, L. R.; Gleriano, J. S \& Chaves, L. D. P. (2019). Contribuições e desafios do gerenciamento de enfermagem hospitalar: evidências científicas. Rev. Gaúcha Enferm; 40: e20180291.

Fukada, M. (2018). Nursing Competency: Definition, Structure and Development. Yonago acta medica, 61(1), 1-7.

Graham, I. D., Logan, J, Harrison, M. B., Straus, S. E., Tetroe, J., Caswell, W., et al. Lost in knowledge translation: time for a map? J Contin Educ Health Prof. 2006; 26(1):13-24. dx.doi:10.1002/chp.47

Oelke, N. D.; Lima, M. A. D. S \& Acosta, A. M. (2015). Translação do conhecimento: traduzindo pesquisa para uso na prática e na formulação de políticas. Rev Gaúcha Enferm; 36(3):113-117.

Oliveira, A. L. G; Silvino, Z. R.; Souza, C. J. (2021). Translation of knowledge in the Implementation of the nursing process in a neonatal unit. Research, Society and Development, 10(8): e23110817263. http://dx.doi.org/10.33448/rsd-v10i8.17263.

Sanna, M. C. (2007). Os processos de trabalho em enfermagem. Rev Bras Enferm; 60(2):221-224.

Silva, Í. R.; Leite, J. L., Trevizan, M. A., Silva, T. P. D. \& Mendes, I. A. C. (2018). Grupos de pesquisa em enfermagem: sistemas complexos para a gestão do conhecimento. REME rev. min. enferm, 22: e-1110.

Tetroe, J. (2007) Knowledge Translation at the Canadian Institutes of Health Research: a primer. Focus Technical Brief;18:1-8.

Vieira, A. C. G, Gastaldo, D \& Harrison, D. (2020). How to translate scientific knowledge into practice? Concepts, models and application. Rev Bras Enferm;73(5):e20190179.

World Health Organization (WHO). (2020). State of the World's Nursing 2020: investing in education, jobs and leadership. Geneva: WHO. www.who.int/publications/i/item/nursing-report-2020.

Yost, J. et al. (2015). The effectiveness of knowledge translation interventions for promoting evidence-informed decision-making among nurses in tertiary care: a systematic review and meta-analysis. Implement Sci; 14(10):98.

Zepeda, K. G. M.; Silva, M. M.; Silva, I. R.; Redko, C. \& Gimbel, S. (2018). Fundamentos da Ciência da Implementação: um curso intensivo sobre um emergente campo de pesquisa. Esc Anna Nery, 22 (2). 